\title{
Meeting the diversity of needs and preferences - a look at the IMS AccessForAll specifications' role in meeting the accessibility agenda efficiently
}

\author{
Martyn Cooper \\ Institute of Educational Technology, The Open University, Walton Hall, Milton Keynes, MK7 6AA, UK \\ m.cooper@open.ac.uk \\ Jutta Treviranus \\ Resource Centre for Academic Technology, Robarts Library, University of Toronto, 130 St. George Street, Toronto, Ontario \\ M5S 3H1, Canada \\ jutta.treviranus@utoronto.ca \\ Andy Heath \\ Communications and Computing Research Centre, Faculty of Arts, Computing, Engineering and Sciences, Sheffield Hallam \\ University, Pond Street, Sheffield, S1 1WB, UK \\ a.k.heath@shu.ac.uk
}

\begin{abstract}
This workshop gives a practical introduction to the IMS AccessForAll specifications that support a new strategy for achieving accessibility to computer-based resources based on the needs and preferences of specific users in the circumstances in which they are operating. This abstract gives a brief introduction to these specifications which will be described by the presenters and used by delegates in the workshop.
\end{abstract}

\begin{abstract}
There are many reasons why a user may have different needs and preferences with respect to their use of a computer, including because they have disabilities. Instead of classifying people by their disabilities, this new approach emphasizes the resulting needs in an information model for formal structured descriptions of them. It then provides a complementary formal structured information model for describing the characteristics of resources required for the matching process. The aim is to make it easy to record this information and to have it in a form that will facilitate the computer system to manage the accessibility provision efficiently.
\end{abstract}

The work described is not about how to create accessible content; that work is done primarily by the World Wide Web Consortium Web Accessibility Initiative (W3C/WAI) [1]. This is outlined for an eLearning context in a publication contributed to by the workshop presenters: the IMS Guidelines for Developing Accessible Learning Applications [2]. The distinguishing feature of the approach described in this workshop is that it provides an approach that combines distributed content into accessible resources and so is not dependent upon the universal accessibility of the original resource.

The specifications, while initiated in the educational community, are suitable for any user in any computer-mediated context. These contexts may include e-government, e-commerce, e-health and more. The specifications can also be used in a number of ways, including: to provide information about how to configure workstations or software applications, to configure the display and control of on-line resources, to search for and retrieve appropriate resources, to help evaluate the suitability of resources for a user, and in the aggregation of resources.

Accessibility, eLearning, user needs, meta-data

\section{ACCESSIBILITY IN A COMPUTER MEDIATED ENVIRONMENT}

Virtually anyone, irrespective of any disability, can be enabled to effectively interact with a computer. Some people with disabilities require alternative access systems, usually referred to as "assistive technology," to enable them to do this and others need the way content is presented to them by the computer to be appropriate or they may need to interact with the computer using methods other than the conventional keyboard and mouse. There are wellestablished principles for how to promote accessibility in software design and electronic content [3, 4]. These 
promote compatibility with assistive technology and ensure that different ways of interacting with the computer can be accommodated.

When activities are computer mediated it is possible to transform how information is presented and controlled. This capability has advanced significantly in the last few years. Many of the necessary technologies are commonly available and it is now relatively easily to customize or personalize network-delivered content and applications. The approach outlined in this workshop depends upon computers to do a lot of the work that increases accessibility. This is a deliberate exploitation of technology. It is consistent with many other uses of technology to manage aspects of publishing and uses the same techniques, including interoperable metadata, or formal structured description strategies and automated transformations.

For a network delivery system to match user needs with the appropriate configuration of a resource, two kinds of descriptions are required: a description of the user's preferences or needs and a description of the resource's relevant characteristics. These two descriptions are the subject of the AccessForAll specifications. The Accessibility for Learner Information Profiles specification (AccLIP) is a specification for describing a user's needs and preferences and the AccessForAll Meta-data specification (AccMD) is a corresponding specification for description of the resource.

Formal descriptions such as the AccLIP and AccMD profiles are known as metadata, simply data about data where the metadata describes the resource or object just as a library catalogue describes a book or journal.

\section{DESCRIBING USER NEEDS AND PREFERENCES}

The specification for user preferences and needs defines a functional description of how a user prefers to have information presented, how they wish to control any function in the application and what supplementary or alternative content they wish to have available. This description is created by users or by their assistants with a simple preference wizard. For example the TILE system's wizard, which will be used in the workshop, asks the following questions when creating a user profile:

What is your preferred language?

Please indicate which of the following apply to you?

I would like to make the text on the screen easier to see.

I would like to enhance the structure of the content.

I would like to enhance the navigation of the content.

I would like alternatives to visual content.

I would like alternatives to textual content.

I would like alternatives to auditory content.

I would like to specify the URL of my personal stylesheet

I would like access to learner scaffolds.

The AccLIP is based on the philosophy that disability is a mismatch between a user's needs and preferences and what they are presented with. It is an artefact of the relationship between a user and an interface or application. Thus a user who is blind does not have a disability in an audio environment but someone who is using a computer without speakers or headphones does. Each user can create a number of AccLIP statements or profiles to accommodate their changing needs within different contexts. A user may have one AccLIP profile for work and another for home if the bandwidth is different, for example. In addition, AccLIP profiles should be able to be changed to suit immediate needs and preferences and to accommodate changes in circumstances or context.

\section{DESCRIBING RESOURCE CHARACTERISTICS: THE CONTENT MODEL}

The AccessForAll approach allows for finer than usual detail with respect to embedded objects and for the replacement of objects within resources where the originals are not suitable on a case-by-case basis. This is made possible by describing the resources in terms of their modalities - auditory, visual, tactile, and text. In addition, the separation between primary and equivalent resources permits flexible dis-aggregation and re-aggregation to meet the individual needs described using AccLIP.

Most resources consist of multiple objects combined into what are commonly known as pages. Sometimes this is done once and there is a static version available and sometimes it is done dynamically for the user. What is unusual about the AccessForAll approach is that the objects that comprise the version of the resource that is sent to the user need not be located in the same place, that is, they may be distributed. In fact, the original composite resource may contain objects that need to be transformed, replaced or augmented; the equivalent objects used for replacing or augmenting may have been created in the original authoring process, or in response to some other user's difficulties with the original resource.

Resources and objects within resources are classified into two categories: primary and equivalent. Most resources are primary resources and require a simple set of statements: how transformable is this resource, what access modality is used (vision, hearing, text literacy or touch) and what is the location of any known equivalent 
alternative. By design, the workload of the creator of the primary materials' metadata is kept as light as possible. Equivalent alternatives such as caption files or image description files are described in their AccMD profile using the same terms as for user preferences in AccLIP profiles. An equivalent object's profile must identify the primary to which it is equivalent. It is expected that the creators of equivalent alternatives and their metadata will be aware of accessibility needs and motivated to provide the details needed to ensure users can locate the equivalent resources.

\section{THE WORKSHOP}

The workshop will provide a practical introduction to this approach. The philosophy and principals will be introduced but the majority of the time will be spent using a system called TILE [5] that implements this so that delegates gain an appreciation of its potential and benefits.

\section{REFERENCES}

[1] WAI Content Guidelines for creating accessible Web pages: http://www.w3.og/TR/WAI-WEBCONTENT/

[2] IMS Guidelines for Developing Accessible Learning Applications, available at: http://wwwr.imsglobal.org/accessibility/accv1p0/imsacc_guidev1p0.html

[3] IMS Guidelines for Developing Accessible Learning Applications, available at: http://www.imsglobal.org/accessibility/accv1p0/imsacc_guidev1p0.html

[4] Cooper M., (2003) Communications and Information Technology (C\&IT) for Disabled Students, in: Powell S. (ed.), Special Teaching in Higher Education- Successful Strategies for Access and Inclusion, (Kogan Page) London 2003

[5] See: http://barrierfree.ca/tile/ 